\title{
MODELO DE DECISIÓN PARA GESTIÓN DE RECURSOS Y PROCESOS EN SISTEMAS DISTRIBUIDOS CON BALANCEO DINÁMICO DE CARGA DE TRABAJO
}

\author{
Jorge Tomás Fornerón Martínez ${ }^{1}$, Federico Agostini² y David Luis La Red Martínez ${ }^{2}$ \\ ${ }^{1}$ Facultad de Ciencias Aplicadas. Universidad Nacional de Pilar. Pilar, Paraguay \\ ${ }^{2}$ Facultad de Cs. Ex. y Naturales y Agrimensura. Universidad Nacional del Nordeste. Corrientes, Argentina
}

\begin{abstract}
RESUMEN
Uno de los problemas que se presentan en los entornos de sistemas distribuidos es que el uso de los recursos compartidos se debe realizar en la modalidad de exclusión mutua, muchas veces con requerimientos de sincronización, consensos para lograr el acceso a grupos de procesos que así lo requieran. Este documento describe cómo se pueden utilizar nuevos modelos de toma de decisiones en grupos de procesos distribuidos que requieren acceso a recursos compartidos en la modalidad de exclusión mutua, incluyéndose un procedimiento para el balanceo de la carga de trabajo.

La aplicación general de los modelos de decisión en los sistemas distribuidos funciona con algoritmos de intercambio de permisos y acuerdos sobre los procesos que intervienen para realizar determinadas acciones.

La creación de un operador de agregación que controle esta asignación de recursos a grupos de procesos y el desarrollo de un modelo de decisión que permita adaptarse a diferentes requerimientos, permitiría asegurar la exclusión mutua, la sincronización y lograría la autorregulación y el balanceo dinámico del sistema. Para satisfacer esto, en cada nodo se debe definir una interfaz entre las aplicaciones y el sistema operativo, que a través de un Runtime (software en tiempo de ejecución complementario al sistema operativo) incluido en dicha interfaz, gestiona los procesos y recursos compartidos y define el escenario correspondiente. Además, los Runtime de los distintos nodos interactúan entre sí para intercambiar información y hay un coordinador global de Runtime en uno de los nodos que evalúa y ejecuta el modelo de decisión y el operador de agregación correspondiente. Como resultado del ciclo de realimentación, y de iteración del modelo de decisión, en base a la actualización continua del estado y de la carga del sistema, se contribuiría al balanceo dinámico y a la autorregulación del sistema.
\end{abstract}

\section{PALABRAS CLAVE}

Modelos de Decisión, Operadores de Agregación, Exclusión Mutua, Sistemas Operativos Distribuidos, Sistemas Autorregulados, Balanceo de Carga

\section{INTRODUCCION}

En los sistemas de telecomunicaciones, existen un conjunto de nodos, separados físicamente, cada uno con sus propias características y recursos heterogéneos, y conforman lo que se denomina Sistema Distribuido, es decir un entorno de trabajo distribuidos para aplicaciones en las cuales los procesos y los recursos que los acceden no están en un mismo lugar, y donde los recursos se deben compartir en la modalidad de exclusión mutua. La implementación de un sistema distribuido se puede realizar sobre cualquier red de comunicaciones, permite la comunicación entre múltiples nodos, y en donde todos los dispositivos forman un solo conjunto de cómputo. Para que esto suceda y para que los nodos que poseen múltiples procesos que cooperan entre sí logren una determinada función, se requiere modelos de decisión que permitan a grupos de procesos utilizar recursos compartidos a los que sólo se puede acceder en la modalidad de exclusión mutua (Tanenbaum, 1996) y (Fitzek y Katz, 2014).

Los métodos considerados tradicionales para este problema se encuentran en (Tanenbaum, 1996 y 2009), donde se describen los principales algoritmos de sincronización en sistemas distribuidos. En (Lodha y Kshemkalyani, 2000), (Cao y Singhal, 2001) y (Ricart y Agrawala, 1981) presentan algoritmos para gestionar la exclusión mutua en redes informáticas. 
En este trabajo se presentará un modelo de decisión, que utilizará un operador de agregación específico para resolver el problema mencionado anteriormente. Esto cae dentro de la categoría de operadores de OWA (Ordered Weighted Average - Promedio Ponderado Ordenado). El uso de operadores de agregación en modelos de decisión ha sido ampliamente estudiado. En (Yager, 1988 y 1993) y (La Red Martínez y Pinto, 2015), se presenta una revisión de los operadores de agregación, especialmente los de la familia OWA.

En este caso, se desarrolla un nuevo modelo de decisión, que utiliza etiquetas lingüísticas con el objetivo de favorecer el acceso de los grupos de procesos a los recursos solicitados. Algunas de las premisas, estructuras de datos y operadores mencionados en (La Red Martínez, 2017) (Agostini et al., 2019) se utilizan como punto de partida para crear un nuevo operador y establecer el modelo de decisión para el escenario mencionado.

\section{MATERIALES Y MÉTODOS}

\subsection{Estructura de Datos}

El objetivo principal de las redes de computadoras es compartir recursos, de tal manera que todos los programas, equipos y datos se encuentren disponibles para quien lo solicite sin importar su ubicación. Para ello se tendrá en cuenta el acceso a recursos compartidos en la modalidad de exclusión mutua distribuida y mediante requisitos de sincronización. Se debe decidir, en función a la demanda de recursos por parte de los procesos, cuáles serán las prioridades para asignar cada uno. Para satisfacer esto, el permiso de acceso a los recursos compartidos de un nodo no sólo dependerá de si los nodos los utilizan o no, sino también del valor de agregación de las preferencias (prioridades) de los distintos nodos en cuanto a la concesión de acceso a los recursos compartidos (alternativas). Los nodos deberán expresar sus prioridades para la asignación de los diferentes recursos compartidos de acuerdo con las necesidades de recursos de cada proceso.

Las premisas, estructuras de datos y el operador mencionado en (La Red Martínez, 2017) y en (Agostini et al., 2019) son aplicadas para resolver el escenario que se describe a continuación. Se operará con lógica difusa, etiquetas lingüísticas y estructuras del tipo 2-tupla, basado en (Dutta et al., 2019).

\subsection{Escenario Propuesto}

Se consideran transiciones de estados de acuerdo a la actualización iterativa de los requerimientos del sistema, en donde se encuentran los distintos nodos, cuyo estado puede ser activo, cuando interactúa con los demás nodos aunque no envíe información ni participe del uso de recursos o la ejecución de procesos con recursos externos, activo compartido, cuando interactúa con los demás nodos, enviando y recibiendo información, creando procesos que utilizan recursos de otros nodos y compartiendo recursos propios con nodos externos, inactivo, cuando no se tiene información del estado real del nodo (puede estar sobrecargado y no puede realizar el intercambio de información, o bien, pudo haberse caído el enlace que lo conecta con los demás nodos), por último, existe un nodo central, que a través de un Runtime gestiona la información de todos los nodos que participen en las asignaciones de recursos. El nodo central, es el encargado de recopilar la información de todos los nodos, aplicar el proceso de agregación y obtener la lista de asignaciones de recursos a procesos. Un macrociclo contempla la iteración de este proceso, en el cual el modelo de decisión es una etapa que se repite $n$ veces. En este macrociclo hay un paso previo de recopilación de información del estado de red, nodos activos y conectados, cuántos están entregando información, un sondeo previo.

\subsection{Operador de Agregación Utilizado por el MD}

En (La Red Martínez, 2017), las características de los operadores de agregación descritos permiten considerar que el método propuesto pertenece a la familia de operadores de agregación Neat-OWA. La propuesta desarrollada en este trabajo consiste en generar un modelo de decisión y su correspondiente operador de agregación para la gestión de grupos de procesos que comparten recursos, realizando modificaciones de los 
operadores de la familia OWA mencionados. Este método es una manera rápida y sencilla para realizar una elección en un problema de decisión.

Para este trabajo lo que se intenta resolver son las solicitudes de recursos por parte de los procesos que se encuentran en nodos distribuidos. Para ello se utilizará la expresión de los valores calculados en términos de 2-tupla utilizando un conjunto de etiquetas lingüísticas. El conjunto de etiquetas utilizado puede variar en cada nodo, es decir, que el conjunto es independiente del nodo central, y es éste el encargado de realizar la traslación lingüística, proceso que consiste en convertir los conjuntos de etiquetas recibidos en el nodo central, en el conjunto que este último trabaja, el cual debe ser el de mayor número de etiquetas. De igual manera, esa traslación lingüística se realiza cuando el nodo central devuelve la información final de prioridades a cada nodo.

En cada nodo se define una interfaz entre las aplicaciones y el sistema operativo, que a través de un Runtime incluido en esa interfaz, gestiona los procesos y recursos compartidos y define el escenario correspondiente. Además, los Runtime interactúan entre sí para intercambiar información y en uno de los nodos hay un Runtime coordinador global que evalúa y ejecuta el modelo de decisión y el operador de agregación correspondiente.

La carga computacional de cada nodo, el número de criterios para determinar la carga de los nodos, los criterios que se aplican y el cálculo de la carga computacional de cada nodo, son los mencionados en (La Red Martínez, 2017). Se debe indicar qué tan importante es para el decisor el criterio en cuestión. Una representación lingüística podría constituirse mediante la ponderación de criterios con la escala de 1 a 5 , y con el siguiente significado: 1) Muy poco satisfactorio 2) Poco satisfactorio 3) Satisfacción media 4) Algo satisfactorio 5) Muy satisfactorio.

Para calcular las prioridades o preferencias de los procesos teniendo en cuenta el estado del nodo, se tiene en cuenta cada solicitud de recursos originada en cada proceso; el cálculo considera el vector de pesos correspondiente según la carga actual del nodo y el vector de los valores concedidos por el nodo según los criterios de evaluación de la solicitud. Los criterios podrían determinarse de acuerdo a la importancia de cada uno, por ejemplo, el significado de cada criterio en una escala de 1 a 9 sería: 1) Extremadamente bajo 2) Muy bajo 3) Bajo 4) Relativamente bajo 5) Medio 6) Relativamente alto 7) Alto 8) Muy alto 9) Extremadamente alto. Los valores son representados en el formato de 2-tupla, considerando las etiquetas lingüísticas propuestas anteriormente. Por lo tanto, el valor de cada criterio deberá compararse con el valor medio que tendrá cada etiqueta, la diferencia mínima de esa comparación será la etiqueta apropiada, algunos de estos conceptos pueden verse en (Dutta et al., 2019).

En resumen, la prioridad nodal (que debe calcularse en el nodo en el que se produce la solicitud) de un proceso de acceso a un recurso determinado (que puede ser en cualquier nodo) se calcula mediante el producto escalar de los vectores mencionados. Esta prioridad nodal debe transformarse en el formato de 2-tuplas, teniendo en cuenta las etiquetas lingüísticas ya mencionadas.

A continuación, es necesario calcular el vector de pesos finales que se utilizará en el proceso de agregación para determinar el orden o la prioridad de acceso a los recursos. El siguiente paso es normalizar los pesos recién obtenidos dividiendo cada uno de ellos por la suma de todos. Así se obtiene un vector de peso normalizado (en el rango de 0 a 1 inclusive) y con la restricción de que la suma de los elementos del vector debe dar 1 . Las prioridades nodales tomadas fila por fila para cada recurso serán multiplicadas escalarmente por el vector de peso final normalizado. De esta manera es posible obtener las prioridades globales finales de acceso de cada proceso a cada recurso. El orden o la prioridad con que se asignarán los recursos y a qué proceso se asignará cada uno, estará determinado por el cálculo de la prioridad global final. La suma de todos los productos en relación con el mismo recurso indicará la prioridad que se le asignará a ese recurso, en relación con los demás recursos que también deberán asignarse. Esto es lo que se denominará Función de Asignación de Sistemas Distribuidos Lingüística (FASDL). Calculando la FASDL para todos los recursos se obtendrá un vector 2-tupla, y ordenando sus elementos de mayor a menor, se obtendrá el orden de prioridad de asignación de recursos. Además, como ya se ha indicado, el mayor de los productos para cada recurso indicará el proceso al que se asignará el recurso. El siguiente paso es repetir el procedimiento, pero eliminando las solicitudes de asignaciones ya realizadas; cabe señalar que los recursos asignados estarán disponibles una vez que los procesos los liberen y, por lo tanto, podrán asignarse a otros procesos. 


\section{MODELO DE DECISIÓN}

El objetivo principal del modelo propuesto es considerar el entorno de ejecución distribuida de procesos, que podrán estar agrupados, con distintas exigencias de consenso respecto del acceso a recursos compartidos, para seleccionar el método de agregación más adecuado a cada escenario posible, para generar la secuencia de asignación de los recursos a los procesos que los solicitan, respetando la exclusión mutua en el acceso a dichos recursos. Este método evalúa la información proveniente de los nodos, para determinar cuál es el escenario según la carga de trabajo proporcionada, y en base a ello elije el operador de agregación correspondiente. En el modelo propuesto, en cada nodo se define una interfaz entre las aplicaciones y el sistema operativo. Comparación de las diferencias más significativas del método propuesto con respecto a los métodos tradicionales, se considera el algoritmo centralizado, el Algoritmo Distribuido de Lamport, Ricart y Agrawala y el Algoritmo de Token Ring (Colouris et al, 1988) y (Tanenbaum y Van Steen, 2001). En cuanto a los requisitos de acceso a recursos el algoritmo propuesto es el único que lo establece calculando prioridades, los demás lo hacen por intercambio de mensajes. El método de asignación en el algoritmo propuesto contempla la mayor prioridad, mientras que en los demás se tiene en cuenta la timestamp más antiguo. Sólo el método propuesto tiene en cuenta los grupos de procesos, mientras que los demás sólo tienen en cuenta procesos independientes. La predisposición (prioridad nodal), carga de nodos, estado nodal (nodos, procesos, grupos, recursos) y estado global del sistema, sólo se tiene en cuenta en el algoritmo propuesto.

\section{Prioridad nodal colaborativa}

En los sistemas distribuidos, se utilizan distintos protocolos de enrutamiento, que permiten la incorporación de nodos a la red (por ejemplo, en una red Ad Hoc). No existe un registro en el sistema sobre la incorporación de un nodo ni de los recursos que este puede ofrecerle a la red, cada nodo puede salir perjudicado en cuanto a cantidad de recursos si ingresa a una red que no llene sus expectativas y que por el contrario sólo le solicite recursos. La red puede estar aceptando un nodo que es egoísta y que solo va a explotar recursos de la red y en realidad no va a aportar nada (Toh et al., 2010) y (Loo et al., 2012). Se implementa un indicador de prioridad por nodo, que depende de la cantidad de recursos y del orden en cual los ofreció. Para el cálculo se utiliza un vector de pesos asociado a orden y cantidad, y es un vector variable, dado que se podría evaluar que se va a ponderar más, si la cantidad de recursos ofrecidos por el nodo, o el orden en el cual los ofrece. Por ejemplo, dos nodos que compartieron la misma cantidad de recursos cada uno, pero uno los compartió de entrada y el otro lo hizo al final, se debería poder balancear ambos criterios, mediante un vector de ponderación en función de la cantidad de recursos y el orden, para obtener un valor de prioridad. Una ponderación sería priorizar de igual manera los dos criterios, el coeficiente sería igual para ambos, también se podría priorizar el orden, por sobre el de la cantidad, o viceversa, por lo cual los valores de coeficientes serían distintos. Como resultado del ciclo de realimentación, se tendría una variable más, actualizada y ponderada, que indicara qué tan colaborativo fue el nodo con los demás, el que fue poco colaborativo va a tener un valor de prioridad colaborativa muy baja, porque habrá tenido muy pocos recursos que ofreció, o porque los ofreció con un orden muy bajo, y otro nodo muy colaborativo va a tener mayor prioridad. Esto contribuye al balanceo dinámico y a la autorregulación del sistema.

\section{CONCLUSION}

\subsection{Evaluación}

Se proporciona una solución global, donde se resalta el dinamismo, la magnitud de los nodos y de los procesos y de la cantidad de requerimientos, que pueden ser aplicables a grandes sistemas, pero también para sistemas de menor cantidad de nodos y requerimientos. Esa variación se explica porque la carga de tráfico, de procesos y de requerimientos varía, sistemas que estaban operando dejan de operar porque terminan y aparecen otros, de pronto se activan nodos con recursos y procesos que hacen falta, los nodos pueden estar activos pero son incorporados al algoritmo cuando algún proceso solicita algún recurso, o algún recurso es solicitado por otro proceso de otro nodo. Un nodo puede estar activo pero no formar parte de las evaluaciones de asignaciones. El modelo propuesto logra establecer un consenso que permite a los grupos de procesos acceder a todos sus recursos de manera secuencial y que éstos no puedan ser removidos hasta que el mismo 
grupo de procesos que los mantiene, los libera. El orden de asignación será determinado por la prioridad promedio general de todas las asignaciones de cada grupo. El sistema distribuido regula y actualiza constantemente el estado local de cada nodo, las decisiones de acceso a los recursos modifican estos estados por lo que debe ser reajustado repetidamente, garantizando la exclusión mutua y reordenando nuevas prioridades. El método debe repetirse siempre que haya grupos de procesos que requieran recursos compartidos.

\subsection{Futuras Líneas de Investigación}

Como trabajo futuro, se prevé desarrollar variantes del método propuesto considerando otros operadores de agregación (especialmente la familia OWA), la consideración de la migración controlada de procesos, con el objetivo de disminuir el tráfico de la red en sistemas distribuidos y la clasificación de grupos de procesadores para la agrupación de nodos según rangos que cumplan ciertas características (velocidad, tiempo de cálculo, procesamiento, etc.).

\section{AGRADECIMIENTOS}

Este trabajo ha sido apoyado por el Proyecto: "Modelos de decisión y operadores de agregación para la gestión de procesos en sistemas distribuidos", código 16F001 de la Universidad Nacional del Nordeste (Argentina).

\section{REFERENCIAS}

Agostini F., Fornerón Martínez J. T., La Red Martínez D. L., 2019. Nuevo operador de agregación para grupos de procesos. $16^{a}$ Conferencia Ibero Americana. Lisboa, Portugal.

Cao G., Singhal M., 2001. A Delay-Optimal Quorum-Based Mutual Exclusion Algorithm for Distributed Systems. IEEE Transactions on Parallel and Distributed Systems. USA .Vol. 12, No. 12, pp 1256-1268.

Colouris G. et al, 1988. Distributed Systems. Concepts and Design. Pearson.

Dutta B., Labella A., Rodríguez R. and Martinez L., 2019. Aggregating Interrelated Attributes in Multi-Attribute Decision-Making With ELICIT Information Based on Bonferroni Mean and Its Variants. International Journal of Computational Intelligence Systems. Vol. 12, pp. 1179. DOI: 10.2991/ijcis.d.190930.002.

Fitzek F. and Katz M., 2014. MOBILE CLOUDS: Exploiting Distributed Resources in Wireless, Mobile and Social Networks. L. John Wiley \& Sons, ed., New Delhi, India. ISBN: 978-0-470-97389-9

La Red Martínez D. L., 2017. Aggregation Operator for Assignment of Resources in Distributed Systems. International Journal of Advanced Computer Science and Applications (IJACSA). The Science and Information (SAI) Organization, England. U.K. Vol. 8, No. 10, pp 406-419, ISSN N² 2156-5570.

La Red Martínez D. L. and Pinto N., 2015. Brief Review of Aggregation Operators. Wulfenia Journal. Austria, Vol. 22, No. 4, pp 114-137.

Lodha S. and Kshemkalyani A., 2000. A Fair Distributed Mutual Exclusion Algorithm. IEEE Trans. Parallel and Distributed Systems. USA. Vol. 11, No. 6, pp 537-549.

Loo J., Lloret Mauri, J., Ortiz J., (2011). Mobile Ad Hoc Networks. Boca Raton: CRC Press. https://doi.org/10.1201/b11447.

Ricart G. and Agrawala A. K., 1981. An Optimal Algorithm for Mutual Exclusion in Computer Networks. Commun. of the ACM, Vol. 24, pp 9-17.

Tanenbaum A. S., 1996. Sistemas Operativos Distribuidos. Prentice - Hall Hispanoamericana S.A., México.

Tanenbaum A. S., 2009. Sistemas Operativos Modernos. 3ra. Edición. Pearson Educación S. A., México.

Tanenbaum A. S., Van Steen M., 2001. Distributed Systems: Principles and Paradigms. Pearson.

Toh C. K., Kim D., Oh S. and Yoo H., 2010. The controversy of Selfish nodes in ad hoc networks. The 12th International Conference on Advanced Communication Technology (ICACT), Phoenix Park. pp. 1087-1092.

Yager R., 1988. On Ordered Weighted Averaging Aggregation Operators in Multi-Criteria Decision Making. IEEE Trans. On Systems, Man and Cybernetics. Vol. 18, No. 1, pp 183-190.

Yager R., 1993. Families of OWA Operators. Fuzzy Sets and Systems. Vol. 59, No. 2, pp 125-148. 